\title{
The Effectiveness of Physical Exercise on Psychological Status, and Sleep Quality among Jordanian Patients Undergoing Hemodialysis: Literature Review
}

\author{
Mutaz Foad Alradaydeh ${ }^{1^{*}(\mathbb{D})}$, Amani Anwar Khalil ${ }^{2}$ \\ ${ }^{1}$ Psychiatric Mental Health Nurse, Health Care Department, The University of Jordan, Amman, Jordan \\ ${ }^{2}$ Clinical Nursing Department, Faculty of Nursing, The University of Jordan, Amman, Jordan \\ Email: *m.foad@ju.edu.jo, a.khalil@ju.edu.jo
}

How to cite this paper: Alradaydeh, M.F. and Khalil, A.A. (2019) The Effectiveness of Physical Exercise on Psychological Status, and Sleep Quality among Jordanian Patients Undergoing Hemodialysis: Literature Review. Open Journal of Nursing, 9, 12671280.

https://doi.org/10.4236/ojn.2019.912092

Received: November 10, 2019

Accepted: December 24, 2019

Published: December 27, 2019

Copyright $\odot 2019$ by author(s) and Scientific Research Publishing Inc. This work is licensed under the Creative Commons Attribution International License (CC BY 4.0).

http://creativecommons.org/licenses/by/4.0/

c) (i) Open Access

\begin{abstract}
Background: Patients receiving hemodialysis are suffered from several psychological complications, and they required to receive safe, feasible, and applicable interventions to improve their psychological health. Practicing physical exercises were used in the literature as a possible intervention to improve patient's outcomes. Purpose: The current literature review aimed to evaluate the effectiveness of physical exercises on psychological status, and sleep quality among patients undergoing hemodialysis, and to detect the gab in the Jordanian literature about the effectiveness of practicing physical exercises on psychological status, and sleep quality among patients undergoing hemodialysis. Methods: The design of the current article was literature review of fifty studies. Results: The current review revealed that most of the research studies were conducted to describe the levels and prevalence's of psychological and mental complication of hemodialysis process, and to evaluate the impact of different types and methods of physical exercises on physical, psychological, and functional outcomes among patients undergoing hemodialysis. However, there are no agreement about the type, time, and intensity of physical exercises, and its impact on patients' psychological outcomes. In Jordanian context, there are no experimental studies were conducted about the effectiveness of physical exercises on psychological status and sleep quality among patients undergoing hemodialysis. Conclusion: It is imperative for researchers to fill the gap that existed in the body of knowledge regarding the effectiveness of physical exercises on psychological outcomes among patients undergoing hemodialysis in order to improve psychological well-being and improve both quality of life for patients, and quality of nursing care.
\end{abstract}




\section{Keywords}

Physical Exercises, Psychological Status, Stress, Anxiety, Depression, Sleep Quality, Hemodialysis

\section{Introduction}

End stage renal disease (ESRD) is a chronic and irreversible stage of kidney failure, which affected more than 430,000 individuals in the United States, and the majority of them (92\%) receiving hemodialysis treatment as renal replacement therapy for 3 times per week, each time prolonged for 4 to 6 hours [1]. In Jordan, the numbers of patients with ESRD increased from 2636 in 2007 to 5352 in 2016 [2], while the numbers of new cases of ESRD for the year 2016 was 829 patients. Most of them (98\%) receiving hemodialysis treatment [2]. The process of hemodialysis causes various physical and functional complications due to the radical changes to their personal life style, recurrent hospitalization, pain, chronic symptoms, dietary limitations, and fear of death [3], which make them highly susceptible for various emotional and psychological consequences [4]. Depression, anxiety, stress, and sleep quality are the most common psychological problems affecting patients undergoing hemodialysis [4].

The prevalence rate of depression among patients receiving hemodialysis is ranging from $20 \%$ to $60 \%$, while the anxiety and stress levels ranging from $21 \%$ to $48 \%$ [4]. Thus, the psychological complications could be deteriorated the physical functioning [5], and sleep quality among patients undergoing hemodialysis [6]. Therefore, there is a prompt needs to evaluate the available ways to improve psychological status (stress, anxiety, and depression, and sleep quality) among patients undergoing hemodialysis.

Practicing physical exercises among hemodialysis patient is considered safe and feasible, and non-pharmacological intervention to improve the physical and psychological functioning [7] [8]. Physical exercise is a bodily movement that is produced by the contraction of skeletal muscles for the upper and lower extremities [9]. However, physical exercise, consists of walking exercises, resistance exercises, and aerobic exercises for the patients' joints according to patients' ability levels [9] [10].

The physical exercise significantly improved the quality of life-mental component scores [11], and reduced the depressive symptoms [12]. However, there are disagreement about the type, time, frequency, and intensity of physical exercises, and it impact on the psychological status, and sleep quality. Therefore, the aims of the current review were to evaluate the effectiveness of physical exercises on psychological status, and sleep quality among patients undergoing hemodialysis, and to detect the gab in the Jordanian literature about the effectiveness of practicing physical exercises on psychological ststus, and sleep quality among patients undergoing hemodialysis. 


\section{Method}

In this review, the searching process was performed by different national and international databases including Google Scholar, Scopus, Web of Science, Science Direct, Pub Med, Med line, CINAHL, EBSCO, Cochrane, in addition to the research gate web site, using the keywords of "physical exercises", "psychological status", "stress", "anxiety”, "depression”, "sleep quality”, and "hemodialysis".

Initially, searching for these key words was performed by the researcher separately for each key word, then a new search was performed while adding a new keyword until including all keywords. Later, the search was performed using all key words in combination with each other. The Boolean operators (AND, OR) have been applied to increase the chance of getting the relevant studies, and to narrow the search results.

The searching process was considered the following inclusion criteria: a) written in English, b) studies used experimental designs such as randomized clinical trial (RCT), uncontrolled trials, and quasi pretest-posttest, and c) published between 2000 and 2019. Articles were excluded from the review if they were a) incomplete reports in the form of editorials, b) opinion papers, and c) conference abstracts. Articles that selected for retrieval were assessed for eligibility criteria and methodological validity by two independent reviewers prior to be included in the review. A total of fifty studies were included in the review.

According to Cochrane guidelines for systemic reviews of interventions, firstly the titles were reviewed. Secondly, abstracts' eligibility was assessed to be included in the review. Then, the available full versions of retrieved studies were reviewed. The excluded studies that used different types of interventions rather than physical exercises, and studies that used different outcomes rather than psychological or sleep related outcomes. Lastly, a PRISMA flowchart was completed to summarize and describe the selection process (Figure 1).

Articles that selected for retrieval were assessed for eligibility criteria and methodological validity by two independent reviewers prior to be included in the review. Critical assessment and review for the eligible articles was done with agreement between the reviewers. Data was extracted from the included articles using standardized data extraction table using specific details about the study design, participants, interventions, and outcomes. Then to display the extracted data, all summarized pages were collected in a one matrix by using word sheet. Any disagreement was resolved through discussion, or with third party judgment if needed.

\section{Results}

\subsection{Physical Exercises among Patients Undergoing Hemodialysis}

Practicing physical exercises among hemodialysis patient is considered safe and feasible non pharmacological treatment to improve the physical and psychological functioning [7] [8]. Physical exercise is a bodily movement that is produced 


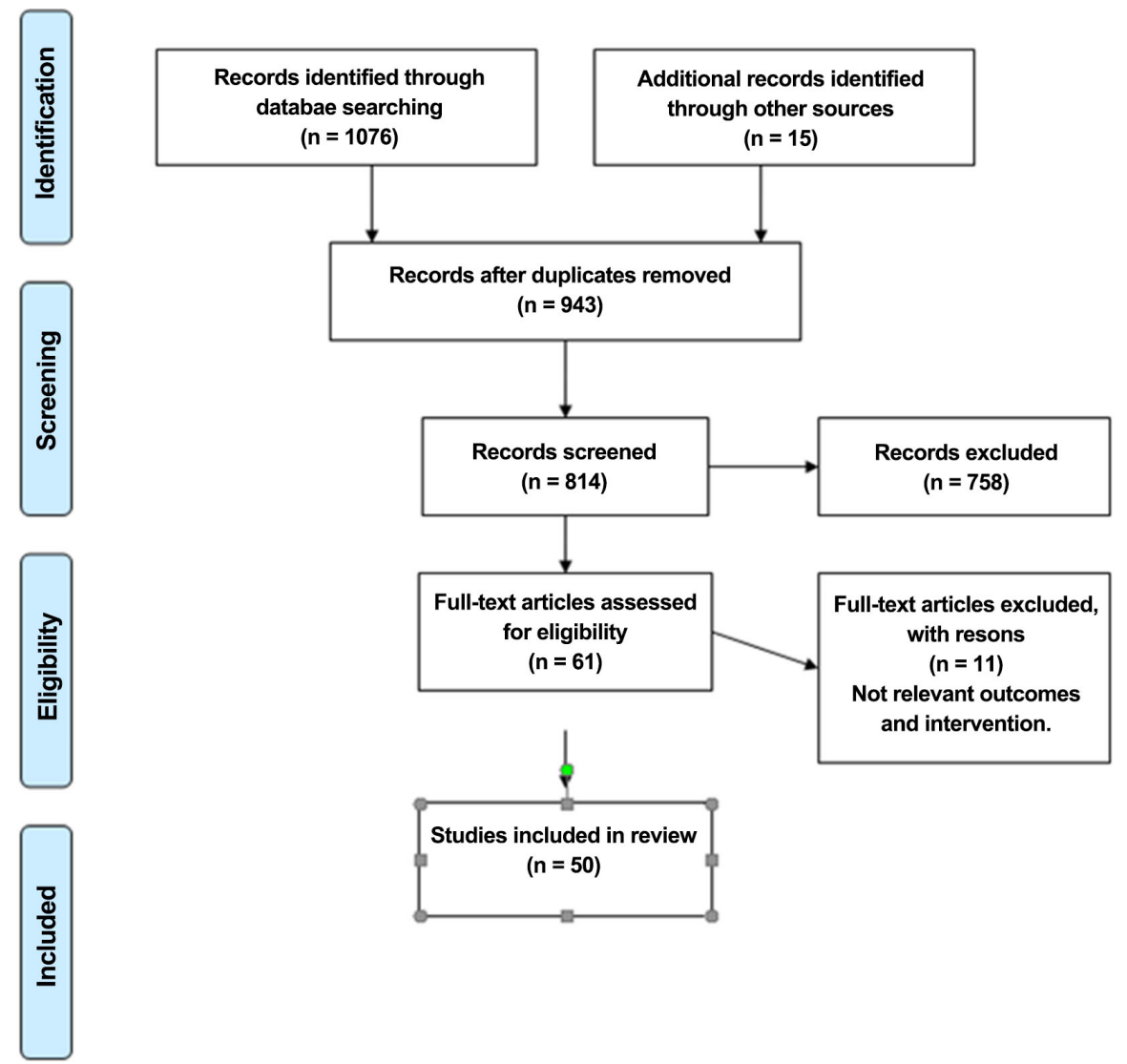

Figure 1. Search strategy and outcomes.

by the contraction of skeletal muscles for the upper and lower extremities [9]. Therefore, Practicing physical exercises were indicated for patients with ESRD to improve the joint flexibility and muscle strength disorders, relive the symptoms of coordination disorders or renal osteopathy, and promote the cardio-vascular stability and reduce the arterial hypertension [13].

Physical exercises among patients with ESRD can be performed during the hemodialysis sessions "intra-dialysis" or between dialysis treatments "extradialysis" at an outpatient health care setting or at fitness centers, or at home [8]. However, the intra-dialysis physical exercise consists of different types of exercises such as warming up exercises, resistance exercises (moving muscle against forces), or aerobic exercises (which increase breathing and heart rate) [14]. While the extra dialysis exercises, also called the inter-dialysis exercises, consists of walking, jogging, bicycling, and stair-stepping [15]. However, practicing physical exercises among patient with ESRD should be started slowly and the intensity, number of repetitions and exercise duration should be gradually increased according to patients' ability levels [9] [10].

The Aerobic physical exercise is an exercise that improves oxygen consumption in the body metabolic or energy generating processes, which includes the range of motion for patients' joints, except the organ which connected to the hemodialysis machine, for a maximum of 15 minutes and during the first two 
hours of dialysis, and based on patients' capacity [16]. These exercises include performing the range of motion or worming exercises for wrist, elbow, shoulder, ankle, knee, and hip joints, applying flexion, extension, abduction, adduction, and rotation (clockwise and counter-clockwise) for each joint [16] [17]. Moreover, the aerobic physical exercise may include special equipment such as using the mini bike equipment, which allows the patients to apply the range of motion exercises for their lower limps for 30-minutes, 3 times a week for 3 months [18].

The resistance physical exercise is a form of strength training in which each effort is performed against specific opposing force generated by resistance (i.e., resistance to being pushed, squeezed, stretched, or bent) [10]. The resistance exercise is used to increase the size and strength of skeletal muscles, and could provide significant functional improvement in overall well-being [19]. The resistance exercise training of the lower limps includes applying ankle weights for knee extension flexion and hip abduction-flexion, the weights can be adjusted in $0.5-1 \mathrm{~kg} /$ week [16]. When patients performed three sets of exercises successfully, the weight could be increased [19]. However, the literature insisted on the importance of stopping the physical exercises (warming and aerobic physical exercises) if the patients feel in any dizziness, headache, palpitations, nausea, exhaustion, or any other adverse effects to protect them of any suspected complications [8].

The current literature showed that both types of physical exercise (warming and aerobic physical exercises) have a positive impact on physical outcomes such as improving inflammatory markers [20], and psychological outcomes such as depression [7]. Furthermore the literature revealed the feasibility and safety for conducting the physical exercises during dialysis session [7] [16]. Moreover, the results of systematic review of clinical trials showed that the intra-dialysis exercises have no significant adverse effects were reported over 30 years of published evidence [8]. In addition, the effectiveness of intra-dialysis exercise programs have been evaluated in many published research articles [21] [22]. The results showed that the exercise during dialysis may have significant impact on physiological, functional, and psychological health [23]. The physical benefits includes promoting solute removal [22], improving oxygen consumption [24], improving dialysis adequacy [25], improving C-reactive protein levels [18], and enhancing serum electrolyte levels (potassium and phosphate) [17]. The functional benefits includes improving physical fitness [25], improving quality of life [26], and sexual functioning [10].

The impact of intra-dialysis physical exercises on the psychological health was reviewed in different studies. Rezaei and Colleagues [27] conducted a study among 51 Iranian participants to assess the effect of regular exercise (warming, stretching, and deep breathing exercises) for ten weeks at home on depression. The results showed significant reduction in depression levels after performing the interventions. Moreover, the levels of anxiety among fourteen hemodialysis patients were significantly improved after performing the warm up, bicycle ergometer, or treadmill exercises in the non-dialysis days, three times weekly for 
12 weeks [24]. In addition, Shimoda and colleagues [12] systematically reviewed the randomized controlled trials studies that investigated the effects of exercise training on depressive symptoms among hemodialysis patients. The results showed that exercise training significantly improved depressive symptoms among the intervention groups as compared with the control groups. The review concluded the need to conduct additional high-quality studies that explore further association between intra-dialysis physical exercises and psychological variables among hemodialysis patients. Furthermore, Salhab and colleagues [11] conducted a systematic review and meta-analysis to examine the effectiveness of physical exercises regarding quality of life, the results concluded significant improvement in the quality of life-mental component scores. However, the results recommended to conduct another types of physical exercises in different geographical areas such as the Middle East, because most of the reviewed studies were conducted in western countries [11].

In Jordan, Darawad and Khalil [28] conducted a correlation study among 190 hemodialysis patients to investigate the patients' perceived exercise benefits and barriers, the results showed that the perceived exercise benefits were significantly higher than the barriers, while the most prospective benefits were preventing muscular atrophy and improving mood. Therefore, these results pointed to the importance to offer specific interventions to improve hemodialysis patients' exercise behaviors. Moreover, around 50\% of Jordanian patients with chronic illnesses have an intentional ability to plan for physical exercise, because they are aware about the benefit and barriers of exercises, while the commitment to exercise plan were significantly correlated with the perceived benefits for the physical exercises $(p>0.05)$ [28] [29]. In addition, the literature concluded that the Jordanian population is physically inactive [30]. However, searching different literature revealed no experimental studies were found about the effectiveness of physical exercises as a coping strategy for patients with chronic illnesses.

The nurses working in hemodialysis unites have the major role in the day-today patient care and management. Therefore, they have a significant role in encouraging and facilitating patients undergoing hemodialysis to improve their physical and psychological health [31]. However, the nephrology nurses work in facilitating the intra-dialysis exercise and maintaining the patients' physical health [32]. On the other hand, there is a gap between the benefits of physical exercise and applying the exercise programs in hemodialysis units [33]. The literature showed that the barriers of implementation an intra-dialysis physical exercise may include different factors such as lack of time, potential exercise-related injury, lack of exercise training skills, and believing that providing intra-dialysis exercise is not nurses' responsibility [32].

\subsection{Physical Exercises and Psychological Status among Patients Undergoing Hemodialysis}

Patients with ESRD and receiving hemodialysis treatments experienced less involvement in social, occupational, and recreational activities, which make them 
had a wide range of somatic and emotional symptoms [34]. The combination of disturbing physical symptoms and psychological complications result in significantly development of depression, which found to be ranged from $20 \%$ to $60 \%$ [4]. This variation could be referred to the measures of depression, location of date collection, and sample size [35]. In Jordan, more than $48 \%$ of the Jordanian patients undergoing hemodialysis treatment had mild to severe depression, and depression were higher among females, married patients, un employed or retired patients, and those with low educational levels [36]. However, the existing results supposed that the prevalence rates of depression among patients undergoing hemodialysis is significantly increasing, and there are prompt needs to find effective strategies to overcome such psychological problem [37].

The prevalence rate of anxiety ranged from $21 \%$ to $48 \%$ [38]. Moreover, the higher levels of anxiety were significantly correlated with females, increased patients' age, low level of education, retirement status, poor financial situation, and co-morbidities [39]. However, the disparity in prevalence's of anxiety reflects the differences in the screening methods and variation in patient's populations [40].

Stresslevelsin patients undergoing hemodialysis were found more than $51 \%$ [41]. In another study, Dumitrescu, Gârneață, and Guzun [42] showed that $60.9 \%$ of hemodialysis patients had moderate to severe stress, and the level of stress were significantly associated with pain, anxiety, age, and income [43]. However, the level of stress varies according to different factors such as gender, educational levels, and employment status [44]. In Jordan, Ahmad and Nazly [45] reported that patients receiving hemodialysis treatment have high levels of psychosocial stress, while, the most common stressors were limitations on time and place for vacation, limitation of fluid intake, and length of dialysis treatment [45].

Physical exercise programs, which include Aerobic, and walking exercises, may have a beneficial effect on mental health in patients with ESRD [4]. The intra-dialysis exercise training program for 10 months will improve the quality of life in both physical functioning and psychological status among patients undergoing hemodialysis treatment [46]. In another study, the exercise training program for one year has a significant impact in reducing the emotional distress among patients with ESRD [47]. Furthermore, providing physical activity on patients undergoing hemodialysis, which consists of elbow flexion/extension, forearm supination/pronation, wrist circumduction, fingers flexion/extension, ankle rotation, toes flexion, extension and hyper extension, were found to be significantly reducing the levels of depression and improving the quality of their life. However, the results recommends to support these finding with other experimental studies using different approach of physical exercises [38].

Shimoda and colleagues [12] conducted a systematic review and meta-analysis of randomized controlled trials that conducted about the effects of supervised exercise on depressive symptoms in hemodialysis patients, the review consisted of only five trials with exercise programs ranged from ten weeks to six months included both intra-dialysis and extra dialysis exercise programs, the results 
showed that exercise training was significantly improve depressive symptoms compared with control groups, however, the systematic review concluded to conduct additional high-quality studies including different ways of intra-dialysis physical exercises to support these findings [12]. Recently, Zhao and colleagues [48] conducted an up-to-date systematic review of the literature to evaluate the effects of exercise on depression among patients with ESRD. The study revealed that exercise can improve depression. However, more studies are needed to confirm the appropriate types of exercise and optimal time for patients with endstage renal disease.

Kouidiand colleagues [47] evaluated the effectiveness of physical exercises such as cycling from 30 to 60 minutes on the levels of anxiety among hemodialysis patients, the results showed that the levels of anxiety was significantly reduced in experimental group than control group. Moreover, a systematic review that conducted about the effectiveness of extra-dialysis physical exercise interventions, the results revealed that exercise intervention is significantly reduces the anxiety levels. However, the review concluded to conduct other type of physical exercises and to confirm the appropriate types of exercise and optimal time for patients with end-stage renal disease [48].

Santhi and colleagues [38] found that regular exercise program, such as performing range of motion exercises for upper and lower joints for the period of 2 months, had significantly reduced the level of stress among 14 patients undergoing hemodialysis. However, searching various data bases, there are no national or international studies were found regarding to the effectiveness of physical exercises on the psychological status among Jordanian population. Moreover, the specific types of physical exercises and optimal time for practicing physical exercises were not confirmed in the literature. Therefore, there is a need to evaluate the effectiveness of physical exercises on the psychological status among Jordanian hemodialysis patients.

\subsection{Physical Exercises and Sleep Quality among Patients Undergoing Hemodialysis}

Patients with end-stage renal disease and undergoing hemodialysis treatment have common disturbances in their sleep quality [49]. The prevalence rates of sleep problems among this population ranges from $50 \%$ to $80 \%$ [50]. Disturbances of sleep quality includes insomnia, restless leg syndrome, sleep disordered breathing, and excessive daytime sleepiness [51]. Several factors may cause disturbances in sleep quality; these factors include pain, dietary restrictions, fatigue, dyspnea, cramps, metabolic abnormalities, peripheral neuropathy, advanced age, and emotional problems [52]. The disturbances of sleep quality were greater among female sex, elderly age, patients with physical or psychological comorbidity, and it was associated with lengthy time on dialysis [51].

Furthermore, Cengic and Colleagues [53] reported that seventy-three percent of patients undergoing hemodialysis had poor sleep quality on weekly basis, of them, the average sleep duration was 4.9 hours, and the average sleep latency 
was $48.2 \mathrm{~min}$, also the results showed that the most common sleep disturbances were insomnia (84.5\%), day and night sleep reversal (39.0\%), and excessive daytime sleepiness (34.0\%).

The sleep disturbances have an important influence on the quality of life [54], and it had been significantly associated with mortalities among patients undergoing hemodialysis [55]. Moreover, disorders of sleep quality are usually associated with behavioral and psychological complications of ESRD. Uzzal [6] showed significant association between depression and Insomnia. Also, the poor sleep quality was greater among patients with high levels of depression [56], and low dialysis adequacy [57]. In Jordan, up to the existing knowledge, there are no available studies described the levels of sleep quality among hemodialysis patients.

While the sleep disorders are common in hemodialysis patients, and it can affect the quality of life and various health outcomes, different research articles tried to find possible ways to improve the sleep quality such as physical exercise. Afshar et al. [38] conducted an experimental study to evaluate the effectiveness of aerobic training exercises on sleep quality among patients with ESRD, the results revealed that sleep quality was improved after 8 weeks in training groups, and the study recommended further research studies about the effectiveness of different kinds of physical exercises. Another study assessed the predictors of sleep quality among hemodialysis patients, the results showed that physical exercise is a predictor for sleep quality [51]. However, the effectiveness of physical exercises on sleep quality levels were not confirmed by the literature, and the appropriate types of exercise and the optimal time for practicing the physical exercises among hemodialysis patients were not clearly articulated in the literature.

\section{Conclusions}

It is clear from the reviewed studies that patients undergoing hemodialysis had several mental and psychological complications which affecting their quality of life and sleep quality. However, the health care providers, including nurses, are responsible for improving the physical, psychological, and functional levels for patients undergoing hemodialysis through encouraging them to practice safe, feasible, and effective interventions. Practicing physical exercises were found to be effective intervention to improve the mental health and quality of life among patients undergoing hemodialysis, while there are no agreement in the reviewed studies about the type, time, frequency, and intensity of physical exercises, and its impact on psychological status (stress, anxiety, and depression), and sleep quality among patients undergoing hemodialysis.

In Jordanian context there are lack of evidence based studies conducted about the levels of psychological status and sleep quality among patients undergoing hemodialysis, and there are no experimental studies evaluated the effectiveness of physical exercises on psychological status and sleep quality among patients undergoing hemodialysis. Therefore, the current review recommends nurses and 
researchers to conduct further research projects in future about the effectiveness of special physical intervention program on several psychological and functional health outcomes.

\section{Acknowledgements}

The researchers thank the University of Jordan and all participants who cooperated with the researchers to take part in this study.

\section{Conflicts of Interest}

All authors declare that there are no conflicts of interest associated with this work.

\section{References}

[1] United States Renal Data System (USRDS) (2004) 2004 Annual Data Report: Atlas of End-Stage Renal Disease in the United States. National Institutes of Health, National Institute of Diabetes and Digestive and Kidney Diseases, Bethesda.

[2] Ministry of Health (2016) Annual Statistical Report of Ministry of Health. https://www.moh.gov.jo/Echobusv3.0/SystemAssets/debff26b-c6df-4244-b7f6-4244 cdf58733.pdf

[3] Deal, B. and Grassley, J.S. (2012) The Lived Experience of Giving Spiritual Care: A Phenomenological Study of Nephrology Nurses Working in Acute and Chronic Hemodialysis Settings. Nephrology Nursing Journal, 39, 471-481.

[4] Wang, L. and Chen, C. (2012) The Psychological Impact of Hemodialysis on Patients with Chronic Renal Failure. In: Polenakovic, M., Ed., Renal Failure-The Facts, IntechOpen, London. https://doi.org/10.5772/36832

[5] Benedetto, M., Lindner, H., Aucote, H., Churcher, J., McKenzie, S., Croning, N. and Jenkins, E. (2014) Co-Morbid Depression and Chronic Illness Related to Coping and Physical and Mental Health Status. Psychology, Health \& Medicine, 19, 253-262. https://doi.org/10.1080/13548506.2013.803135

[6] Uzzal, O.K., Islam, M.N., Ahmed, P.I., Mamun, M.A., Hossain, M.B., Bhuiyan, F.K. and Khan, M.F. (2015) Depression and Insomnia in Patients on Maintenance Hemodialysis. Journal Dhaka Medical Collage, 24, 3-11.

https://doi.org/10.3329/jdmc.v24i1.29555

[7] Chung, Y.C., Yeh, M.L. and Liu, Y.M. (2016) Effects of Intradialytic Exercise on the Physical Function, Depression and Quality of Life for Haemodialysis Patients: A Systematic Review and Meta-Analysis of Randomised Controlled Trials. Journal of Clinical Nursing, 26, 1801-1813. https://doi.org/10.1111/jocn.13514

[8] Ma, S., Lui, J., Brooks, D. and Parsons, T. (2012) The Availability of Exercise Rehabilitation Programs in Hemodialysis Centers in Ontario. CANNT Journal, 22, 28-36.

[9] Painter, P. (2005) Physical Functioning in End-Stage Renal Disease Patients: Update 2005. Hemodilaysis International, 9, 218-235. https://doi.org/10.1111/j.1492-7535.2005.01136.x

[10] Lima, M., Cicotoste, C., Cardoso, K., Junior, L., Monteiro, M. and Dias, A. (2013) Effect of Exercise Performed during Hemodialysis: Strength versus Aerobic. Renal Failure, 35, 697-704. https://doi.org/10.3109/0886022X.2013.780977

[11] Salhab, N., Karavetian, M., Kooman, J., Fiaccadori, E. and El Khoury, C. (2018) Ef- 
fects of Intradialytic Aerobic Exercise on Hemodialysis Patients: A Systematic Review and Meta-Analysis. Journal of Nephrology, 32, 549-566.

https://doi.org/10.1007/s40620-018-00565-Z

[12] Shimoda, T., Matsuzawa, R., Hoshi, K., Yoneki, K., Harada, M., Watanabe, T. and Matsunaga, A. (2017) Effects of Supervised Exercise on Depressive Symptoms in Hemodialysis Patients: A Systematic Review and Meta-Analysis of Randomized Controlled Trials. Renal Replacement Therapy, 3, 56. https://doi.org/10.1186/s41100-017-0136-5

[13] Mahrova, A. and Svagrova, K. (2013) Exercise Therapy-Additional Tool for Managing Physical and Psychological Problems on Hemodialysis. In: Suzuki, H., Ed., Hemodialysis, IntechOpen, London. https://doi.org/10.5772/53058

[14] Koh, K.P., Fassett, R.G., Sharman, J.E., Coombes, J.S. and Williams, A.D. (2010) Effect of Intradialytic versus Home-Based Aerobic Exercise Training on Physical Function and Vascular Parameters in Hemodialysis Patients: A Randomized Pilot Study. American Journal Kidney Disease, 55, 88-99. https://doi.org/10.1053/j.ajkd.2009.09.025

[15] Painter, P., Blagg, C. and Moore, G. (1995) Exercise for the Dialysis Patient: A Prescription Guide. The Life Options Rehabilitation Advisory Council. Medical Media Associates.

[16] Cheema, B., O’sullivan, A., Chan, M., Patwardhan, A., Kelly, J., Gillin, A. and Fiataronesingh, M. (2006) Progressive Resistance Training during Hemodialysis: Rationale and Method of a Randomized-Controlled Trial. Hemodialysis International, 10, 303-310. https://doi.org/10.1111/j.1542-4758.2006.00112.x

[17] Makhlough, A., Ilali, E., Mohseni, R. and Shahmohammadi, S. (2012) Effect of Intra-Dialytic Aerobic Exercise on Serum Electrolytes Levels in Hemodialysis Patients. International Journal of Kidney Disease, 6, 119-123.

[18] Afshar, R., Emany, A., Saremi, A., Shavandi, N. and Sanavi, S. (2011) Effects of Intradialytic Aerobic Training on Sleep Quality in Hemodialysis Patients. Iranian Journal of Kidney Diseases, 5, 119-123.

[19] Afshar, R., Shegarfy, L., Shavandi, N. and Sanavi, S. (2010) Effects of Aerobic Exercise and Resistance Training on Lipid Profiles and Inflammation Status in Patients on Maintenance Hemodialysis. Indian Journal of Nephrology, 20, 185-191. https://doi.org/10.4103/0971-4065.73442

[20] Cruz, L., Zanetti, H., Andaki, A., Mota, Neto, O. and Mendes, E. (2018) Intradialytic Aerobic Training Improves Inflammatory Markers in Patients with Chronic Kidney Disease: A Randomized Clinical Trial. Universidade Estadual Paulista, 24, 1-5. https://doi.org/10.1590/s1980-657420180003e017517

[21] Segura-Ortí, E. and Johansen, K.L. (2010) Exercise in End-Stage Renal Disease. Seminars in Dialysis, 23, 422-430. https://doi.org/10.1111/j.1525-139X.2010.00766.x

[22] Parsons, T.L., Toffelmire, E. and King-VanVlack, C. (2006) Exercise Training during Hemodialysis Improves Dialysis Efficacy and Physical Performance. Archives of Physical Medicine and Rehabilitation, 87, 680-687.

https://doi.org/10.1016/j.apmr.2005.12.044

[23] Cheema, B., Smith, B. and Fiatarone Singh, M.A. (2005) Rationale for Intradialytic Exercise Training as Standard Clinical Practice in End Stage Renal Disease. American Journal of Kidney Disease, 45, 912-916. https://doi.org/10.1053/j.ajkd.2005.01.030

[24] Suh, M., Jung, H., Kim, S. and Park, J. (2002) Effects of Regular Exercise on Anxiety, Depression, and Quality of Life in Maintenance Hemodialysis Patients. Renal 
Failure, 24, 337-345. https://doi.org/10.1081/JDI-120005367

[25] Bayoumi, M. and Alwakeel, J. (2015) Impacts of Exercise Programs on Hemodialysis Patients' Quality of Life and Physical Fitness. Quality in Primary Care, 23, 192-200.

[26] Neto, M., Lacerda, F., Lopes, A., Martinez, B. and Saquetto, M. (2018) Intradialytic Exercise Training Modalities on Physical Functioning and Health-Related Quality of Life in Patients Undergoing Maintenance Hemodialysis: Systematic Review and Meta-Analysis. Clinical Rehabilitation, 32, 1189-1202. https://doi.org/10.1177/0269215518760380

[27] Rezaei, J., Abdi, A., Rezaei, M., Heydarnezhadian, J. and Jalali, R. (2015) Effect of Regular Exercise Program on Depression in Hemodialysis Patients. International Scholarly Research Notices, 2015, Article ID: 182030. https://doi.org/10.1155/2015/182030

[28] Darawad, M. and Khalil, A. (2013) Jordanian Dialysis Patients' Perceived Exercise Benefits and Barriers: A Correlation Study. Rehabilitation Nursing, 38, 315-322. https://doi.org/10.1002/rnj.98

[29] Darawad, M., Khalil, A., Hamdan-Mansour, A. and Nofal, B. (2014) Perceived Exercise Self-Efficacy, Benefits and Barriers, and Commitment to a Plan for Exercise among Jordanians with Chronic Illnesses. Rehabilitation Nursing, 41, 342-351.

[30] Ammouri, A., Neuberger, G., Nashwan, A. and Al-haj, A. (2007) Determinants of Self-Reported Physical Activity among Jordanian Adults. Journal of Nursing Scholarship, 39, 342-348. https://doi.org/10.1111/j.1547-5069.2007.00191.x

[31] Bennett, P.N. (2011) Technological Intimacy in Haemodialysis Nursing. Nursing Inquiry, 18, 247-252. https://doi.org/10.1111/j.1440-1800.2011.00537.x

[32] Bennett, P.N., Peter, J., Wang, W. and Street, M. (2016) Attitudes of Nephrology Nurses towards Patient Exercise during Hemodialysis. Nephrology Nursing Journal, 43, 331-337.

[33] Greenwood, S.A., Koufaki, P., Rush, R., Macdougall, I.C. and Mercer, T.H. (2014) Exercise Counselling Practices for Patients with Chronic Kidney Disease in the UK: A Renal Multidisciplinary Team Perspective. Nephron Clinical Practice, 128, 67-72. https://doi.org/10.1159/000363453

[34] Murtagh, F.E., Addington-Hall, J. and Higginson, I.J. (2007) The Prevalence of Symptoms in End-Stage Renal Disease: A Systematic Review. Advanced Chronic Kidney Disease, 14, 82-99. https://doi.org/10.1053/j.ackd.2006.10.001

[35] Watnick, S., Wang, P.L., Demadura, T. and Ganzini, L. (2005) Validation of Two Depression Screening Tools in Dialysis Patients. American Journal of Kidney Disease, 46, 919-924. https://doi.org/10.1053/j.ajkd.2005.08.006

[36] Alradaydeh, M.F. and Khalil, A.A. (2017) The Association of Spiritual Well-Being and Depression among Patients Receiving Hemodialysis. Perspective of Psychiatric Care, 54, 341-347.

[37] Geraldo, S., Elizabeth, D., Ana Paula, B., Rafael, L., Mikaelly, L., Eveline, S., Aline, S. and JoãoMoisés, S. (2014) Depression among Patients with End-Stage Renal Disease in Hemodialysis. Psychology, Health \& Medicine, 19, 547-551. https://doi.org/10.1080/13548506.2013.845303

[38] Santhi, A., Samson, R. and Pethuru, D. (2018) Effectiveness of Physical Activity on Depression, Anxiety, Stress and Quality of Life of Patients on Hemodialysis. Biomedical Research, 29, 1885-1890.

https://doi.org/10.4066/biomedicalresearch.29-18-177

[39] Gerogianni, G., Lianos, E., Kouzoupis, A., Polikandrioti, M. and Grapsa, E. (2018) 
The Role of Socio-Demographic Factors in Depression and Anxiety of Patients on Hemodialysis: An Observational Cross Sectional Study. International Urology and Nephrology, 50, 143-154. https://doi.org/10.1007/s11255-017-1738-0

[40] Cohen, S., Cukor, D. and Kimmel, P. (2016) Anxiety in Patients Treated with Hemodialysis. Clinical Journal American Social Nephrology, 11, 2250-2255.

[41] HeidariGorji, A., Davanloo, A. and Heidarigorji, A.M. (2014) The Efficacy of Relaxation Training on Stress, Anxiety, and Pain Perception in Hemodialysis Patients. Indian Journal Nephrology, 24, 356-361. https://doi.org/10.4103/0971-4065.132998

[42] Dumitrescu, A.L., Gârneață, L. and Guzun, O. (2009) Anxiety, Stress, Depression, Oral Health Status and Behaviours in Romanian Hemodialysis Patients. Romanian Journal of Internal Medicine, 47, 161-168.

[43] Gorji, M., Davanloo, A. and Heidarigorji, A. (2014) The Efficacy of Relaxation Training on Stress, Anxiety, and Pain Perception in Hemodialysis Patients. Indian Journal of Nephrology, 24, 6. https://doi.org/10.4103/0971-4065.132998

[44] Quinan, P. (2007) Control and Coping for Individuals with End Stage Renal Disease on Hemodialysis: A Position Paper. CANNT Journal, 17, 77-84.

[45] Ahmad, M. and Nazly, E. (2014) Hemodialysis: Stressors and Coping Strategies. Psychology, Health \& Medicine, 20, 477-487. https://doi.org/10.1080/13548506.2014.952239

[46] Ouzouni, S., Kouidi, E., Sioulis, A., Grekas, D. and Deligiannis, A. (2009) Effects of Intradialytic Exercise Training on Health-Related Quality of Life Indices in Hemodialysis Patients. Clinical Rehabilitation, 23, 53-63.

https://doi.org/10.1177/0269215508096760

[47] Kouidi, E., Karagiannis, V., Grekas, D., Iakovides, A., Kaprinis, G., Tourkantonis, A., et al. (2010) Depression, Heart Rate Variability, and Exercise Training in Dialysis Patients. European Journal Cardiovascular Rehabilitation, 17, 160-167. https://doi.org/10.1097/HJR.0b013e32833188c4

[48] Zhao, Q., Zhang, H., Wen, X., Wang, Y., Chen, X., Chen, N., Sun, Y., Liu, H. and Lu, P. (2019) Exercise Interventions on Patients with End-Stage Renal Disease: A Systematic Review. Clinical Rehabilitation, 33, 147-156. https://doi.org/10.1177/0269215518817083

[49] Parker, K.P. (2003) Sleep Disturbances in Dialysis Patients. Sleep Medicine Review, 7, 131-143. https://doi.org/10.1053/smrv.2001.0240

[50] Iliescu, E.A., Coo, H. and McMurray, M.H. (2003) Quality of Sleep and Health-Related Quality of Life in Haemodialysis Patients. Nephrology Dialysis Transplantation, 18, 126-132. https://doi.org/10.1093/ndt/18.1.126

[51] Guney, I., Atalay, H., Solak, Y., Altintepe, L., Toy, H., Tonbul, Z. and Turk, S. (2010) Predictors of Sleep Quality in Hemodialysis Patients. International Journal of Artificial Organs, 33, 154-160. https://doi.org/10.1177/039139881003300304

[52] Kusleikaite, N., Bumblyte, I.A., Razukeviciene, L., Sedlickaite, D. and Rinkunas, K. (2005) Sleep Disorders and Quality of Life in Patients on Hemodialysis. Medicina (Kaunas), 41, 69-74.

[53] Cengic, B., Resic, H., Spasovski, G., Avdic, E. and Alajbegovic, A. (2012) Quality of Sleep in Patients Undergoing Hemodialysis. International Urology Nephrology, 44, 557-567. https://doi.org/10.1007/s11255-010-9881-x

[54] Wlliams, S.W., Tell, G.S., Zheng, B., Shumaker, S., Rocco, M.V. and Sevick, M.A. (2002) Correlates of Sleep Behavior among Hemodialysis Patients. American Journal Nephrology, 22, 18-28. https://doi.org/10.1159/000046670

[55] Benz, R.L., Pressman, M.R., Hovick, E.T. and Peterson, D.D. (2000) Potential Novel 
Predictors of Mortality in End-Stage Renal Disease Patients with Sleep Disorders. American Journal Kidney Disorder, 35, 1052-1060. https://doi.org/10.1016/S0272-6386(00)70039-4

[56] Pai, M., Hsu, S., Yang, S., Ho, T., Lai, C. and Peng, Y. (2007) Sleep Disturbance in Chronic Hemodialysis Patients: The Impact of Depression and Anemia. Renal Failure, 29, 673-677. https://doi.org/10.1080/08860220701459642

[57] Trbojević-Stanković, J., Stojimirović, B., Bukumirić, Z., Hadžibulić, E., Andrić, B., Djordjević, V., Marjanović, Z., Birdjozlić, F., Nešić, D. and Jovanović, D. (2014) Depression and Quality of Sleep in Maintenance Hemodialysis Patients. Serbian Archives of Medicine, 142, 437-443. https://doi.org/10.2298/SARH1408437T 